\title{
Variation of large elastodynamic earthquakes on complex fault systems
}

\author{
Bruce E. Shaw \\ Lamont-Doherty Earth Observatory, Columbia University, Palisades, New York, USA
}

Received 10 March 2004; revised 14 June 2004; accepted 4 August 2004; published 29 September 2004.

[1] One of the biggest assumptions, and a source of some of the biggest uncertainties in earthquake hazard estimation is the role of fault segmentation in controlling large earthquake ruptures. Here we apply a new model which produces sequences of elastodynamic earthquake events on complex segmented fault systems, and use these simulations to quantify the variation of large events. We find a number of important systematic effects of segment geometry on the slip variation and the repeat time variation of large events, including an increase in variation at the ends of segments and a decrease in variation for the longest segments. INDEX TERMS: 7209 Seismology: Earthquake dynamics and mechanics; 7221 Seismology: Paleoseismology; 7223 Seismology: Seismic hazard assessment and prediction; 7260 Seismology: Theory and modeling; 8010 Structural Geology: Fractures and faults. Citation: Shaw, B. E. (2004), Variation of large elastodynamic earthquakes on complex fault systems, Geophys. Res. Lett., 31, L18609, doi:10.1029/2004GL019943.

\section{Introduction}

[2] The faults on which earthquake occur are not simple planar structures, but have bends, splays, and steps in them. These geometrical features are used to define segments of faults, which are themselves used to delineate future expected large events. The role of fault segmentation in determining future large earthquakes is not, however, well understood. While there are many instances of large earthquakes initiating and terminating at geometrical discontinuities [King and Nabelek, 1985], there are also examples such as the 1992 M7.1 Landers events which jumped two segment stepovers and then died in the middle of a third segment. Underlying these complications is the long repeat times of large earthquakes - of order hundreds of yearswhich make simple observational answers hard to find. Despite the limited observations, current planning efforts for future earthquakes revolve centrally around the concept of fault segmentation, defining fault segments and then relying on panels of experts to vote on which segments they think might break separately or together [Working Group on California Earthquake Probabilities, 2002]. Clearly, there is a need for more scientific understanding of this problem.

[3] Improvements in our understanding of the physics operating on various timescales has allowed improvements on our ability to do time dependent hazard estimation [Dieterich, 1994; Parsons et al., 2000]. On long timescales used for planning and mitigation purposes (e.g., the 50 year probabilities used in the national hazard maps), a critical parameter affecting these hazard estimates is the coefficient

Copyright 2004 by the American Geophysical Union. 0094-8276/04/2004GL019943\$05.00 of variation of large event repeat times (the standard deviation of the repeat times divided by the mean repeat time). For large coefficients of variation there is little change in the probabilities of large events occurring during the earthquake cycle, and the time dependence of long term probabilities become negligible. In contrast, for smaller coefficients of variation, the distribution approaches a periodic distribution, we have more pronounced changes in the probabilities during the earthquake cycle, and the potential of doing time dependent long term hazard estimation becomes significant. What the appropriate value or values of the coefficient of variation are for earthquakes remains a hotly debated topic, with major implications for earthquake predictability and hazard estimates [Working Group on California Earthquake Probabilities, 2002; Lindh, 2003].

[4] Fueling the controversy is the paucity of observational data from which values can be obtained. Important constraints have been derived from direct observations of the time intervals between the few areas with historical records [Nishenko and Buland, 1987; Lindh, 2003; Sykes, 2003]. There are, however, a number of limitations with this approach, including the small number of events in each sequence, and thus the need to average over widely different fault systems, and the long times between large eventshundreds of years - which precludes much additional improvements in the data.

[5] Other observational contributions have come from paleoseismic trenches, which record sequences of ruptures at individual points along a fault. Trenches, however, have yielded only limited sequence lengths, and concerns about missing events, which may be difficult to see or may have ruptured nearby branches, further complicate these efforts. For perhaps the best recorded site, where a remarkable 14 events have been dated at Wrightwood [Fumal et al., 2002] a further issue complicates a simple interpretation of the data: it has been argued that the site may be near an overlap of large events rupturing to the north and to the south, and thus the relatively large coefficient of variation measured there is not typical of values along the length of fault. With these observational limitations, and the difficulty of obtaining further data, other approaches which can contribute to this problem are obviously needed.

[6] Here, we present numerical results from a newly developed model which generates long sequences of elastodynamic events on complex fault systems [Shaw, 2004]. The model has a number of features which are important to bring to bear on this problem. First, it self-consistently generates a complex fault system geometry, through a physical mechanism rather than being externally imposed. This self-consistency is important in insuring strain is compatibly accommodated in the long run over many earthquake cycles. The self-consistency also reduces the 

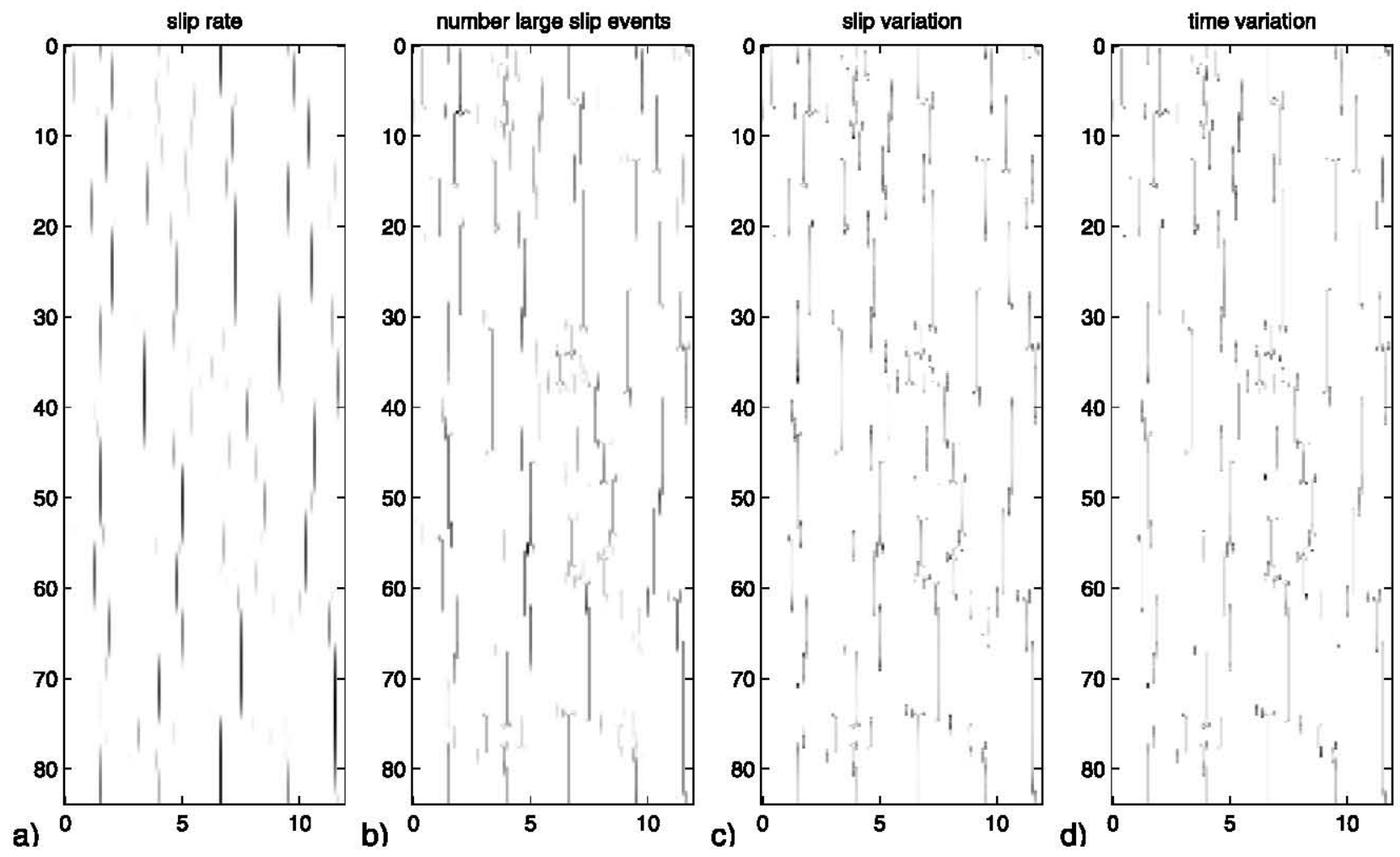

Figure 1. Different measures of a long sequence of dynamic events on a complex fault system. The simulation was run so that we have typically tens of large event cycles occurring on each fault. Axes are distances in units of the seismogenic layer depth. a) Slip rate on faults, including all events large and small. b) Number of large events during which a point on fault slips. c) Slip variation of large events. d) Time variation of large events.

number of things which must be specified, by allowing the fault system to self-organize from a simple physics, here a random strength heterogeneity combined with a long term slip weakening. The complex geometry is important in the ability to study the role of fault geometry in the problem, particularly since fault segmentation is a foundation upon which seismic hazard maps are based. Second, it selfconsistently generates sequences of elastodynamic events on the fault system. The long sequences are critical here in that the stresses left over by previous events form the setting for subsequent events. The self-consistency and elastodynamics are important in our ability to study the interaction of geometry and dynamics and to simulate the cascading ruptures seen, as studies of individual ruptures on segmented faults have illuminated the critical role of the prestress in the ability of ruptures to jump stepovers [Harris et al., 1991]; here the sequences generate their own distributions of prestress. Finally, our ability to simulate long sequences of events allows us not only to reach a representative population of events, the attractor of the dynamics, but also to examine statistical measures of the system over the timescale of many many earthquake cycles, to thus elucidate quantitative measures relating dynamics, geometry, and the variation of large events.

\section{The Model}

[7] The model geometry is meant to capture the behavior of an extensional fault system like the Basin and Range in the Western U.S. The model consists of a scalar two dimensional brittle upper layer coupled to a slowly stretching ductile substrate. When the stresses in the brittle layer exceed the strength, dislocations occur. All of the nonlinearity in the problem comes from how the strength evolves. It begins from some initial unbroken strength having some overall value plus a spatially random component. A long term geological slip weakening localizes the slip onto faults and leads to a slow geological evolution of the fault system [Spyropoulos et al., 2002]. Dynamic weakening during slip events leads to sudden stick-slip events. A variety of dynamic weakening mechanisms are explored, including slip-weakening, velocity-weakening, and time-weakening [Shaw, 1997; Shaw and Rice, 2000]. We explore a range of frictions because the frictional behavior at seismic slip rates remains a fundamental open question. The model, and the equations defining it have been presented elsewhere [Shaw, 2004]; we include them for completeness as auxiliary material ${ }^{1}$. Except where noted, we use slip-weakening for the dynamic weakening. Lengths in the problem are scaled to the seismogenic depth, which has been scaled to unity.

\section{Results}

[8] Beginning from some stage in the slow geological evolution of the fault system, we examine a long sequence of elastodynamic ruptures. These ruptures display a rich

\footnotetext{
${ }^{1}$ Auxiliary material is available at $\mathrm{ftp} / / / \mathrm{ftp}$. agu.org/apend/gl/ 2004GL019943.
} 


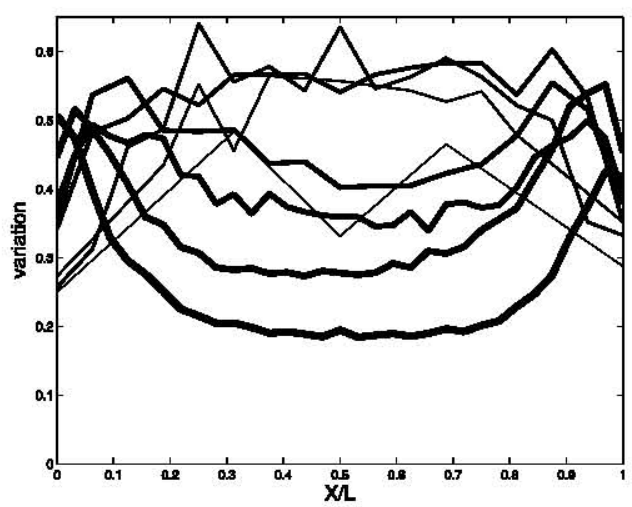

Figure 2. Slip variation along segments. Thicker lines indicate longer segments. Length bins are between $L=.33$, $.56, .92,1.5,2.5,4.1,6.8,11.2$.

complexity of behaviors. Figure 1 shows a number of different ways of looking at a catalogue of events which have occurred on the fault system. Figure 1a shows with a greyscale the slip rate on the faults. We see the major faults most prominently in this view. We also see that the longest faults tend to slip the fastest. Figure $1 \mathrm{~b}$ shows the number of times a part of the fault has slipped in a large event. This highlights the most active areas, and also shows more of the smaller fault segment features which, though slipping less during large events, nevertheless do break during the large events and help accommodate slip on the fault system. Large events are defined by events which break a total length of faults greater than the seismogenic depth of unity. The numerous small events are not considered in the statistical analysis in this paper so that we can focus on the variation of large events. A segment here is defined as a straight continuously broken length of fault; stepovers mark places where segments link to form larger faults. In the various plots which follow, tens of repeat times are used, so small catalogue lengths are not an issue [Working Group on California Earthquake Probabilities, 2002; Stein and Newman, 2004].

[9] The final two panels show the core of this paper's results. Figure $1 \mathrm{c}$ shows the variation in slip of the large events. We see a number of interesting things in this plot. First, at least on the largest main segments, the larger variation is tending to happen at the ends of the segments relative to the middle (e.g., the fault centered near 3 in the horizontal spanning around 32 to 43 in the vertical). Second, the largest segments appear to typically have less overall variability than the smaller segments.

[10] The final panel, Figure 1d, shows the variation in recurrence time of the large events. Because of its central significance in seismic hazard, this is the key measure. It is, furthermore, illuminating of some interesting dynamics. In Figure 1d we see both of the features mentioned regarding Figure 1c, that the ends of the largest segments tend to be more variable than the middles, and that the larger segments are less variable.

[11] We can make all of these statements more quantitative by averaging the variability over segments. To do this, we first group segments of similar length. We then normalize the horizontal axis by the segment length, and average over the group of segments of similar length. In Figure 2 we plot the results of the slip variation averaged in this way,

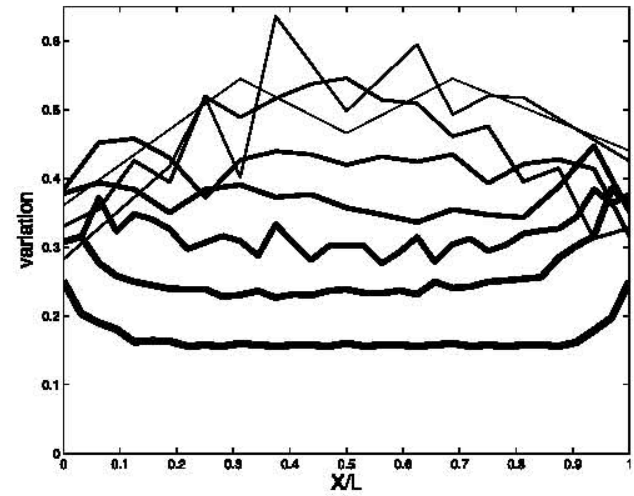

Figure 3. Time variation along segments. Thicker lines indicate longer segments.

with thicker lines corresponding to longer segment lengths. The main features mentioned before are clearly shown: for the thickest lines indicating the longest segments, the variation is larger at the ends compared to the middle, and the average variation across the segment length of the thickest lines is the lowest. An additional feature which can be seen is a qualitative contrast of the smaller segments, which actually show higher variability in their centers as compared with their ends.

[12] Figure 3 shows the same averaging as in Figure 2, only now with the time variation. As before, the ends of the largest segments show higher variation than the centers, and the largest segments have the lowest overall variation. We also see here that the time variation is quantitatively different than the slip variation, being in particular somewhat lower for the time versus slip variation. There is also an interesting qualitative difference: whereas the slip variation has a spatial dependence along the segment length which scales with the segment length, the time variation has a spatial dependence along the segment length which scales with the seismogenic depth elastic length. (The steepening of the time variation near the segment ends for long segments indicates this, and a plot similar to Figure 3 but

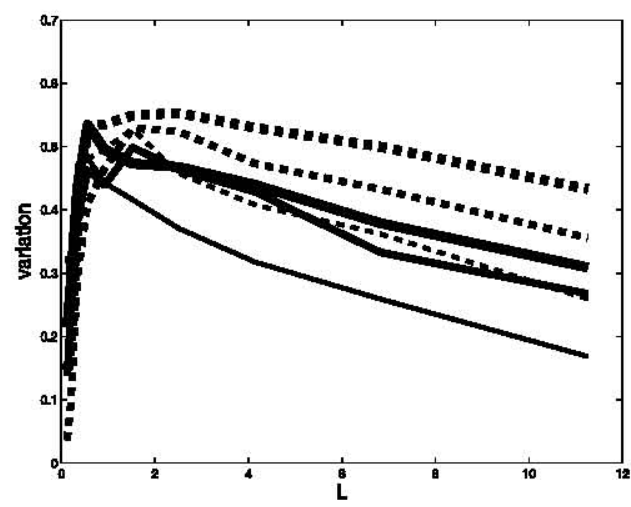

Figure 4. Average time variation and space variation as a function of segment length, for different frictional instabilities. Time variation is solid line, while slip variation is dashed line. Thinnest lines are slip weakening, thickest lines are velocity weakening, and intermediate thickness lines are time weakening. Note for all frictional instabilities the time variation is less than the slip variation for the longest segments. 
with the horizontal axis unscaled by segment length shows this most clearly). Thus the slip variation and time variation are not simple proxies for each other.

[13] The last figure, Figure 4, shows averages of variation as a function of segment length, for different frictional instabilities. We average the information in Figures 2 and 3 along the segment lengths, and then plot the average variation as a function of segment length. We do this on the same fault system using three different frictional instabilities, plotting the time variation with solid lines and the slip variation with dotted lines. The thin lines show a slip weakening friction, as was used in the previous figures. The somewhat thicker lines show a time weakening friction. The thickest lines show a velocity-weakening friction. For all of the frictions, we see a maximum variability around the elastic lengthscale of unity and a decrease in variability for the longest segments. We also see that the slip variability is larger than the time variability for the long segments. The velocity weakening friction shows the highest variability, followed, interestingly, by the time-weakening and then the slip-weakening. Clearly, geometry and dynamics are both playing a role in quantitatively determining the results. Nevertheless, the common qualitative features we have found allow us to extract useful information even in the absence of a settled understanding of the friction on faults.

\section{Implications}

[14] The significant systematic effect we have seen in large event variability along segments, and with segments lengths has a number of important implications for seismic hazard estimation. First, if the Wrightwood paleoseismic trench is indeed near a segment boundary, the high values of variation measured there may not be typical of other parts of the San Andreas. A comparison with values more in the center of segments would be extremely valuable. Second, we find important differences between slip variation and time variation, so slip variability is not a sufficient proxy for time variability; it is, however, seen to bound the time variability for the longest segments. Third, we find good news for time dependent hazard estimation, in that the largest segments and largest events appear to be the most regular. More sophisticated hazard estimates could incorporate this change in the time variability with event size. Finally, older fault systems with smoother longer fault segments may be more regular than younger fault systems; global data averaging across varying faults [Nishenko and Buland, 1987] may need to take this into account.

[15] Acknowledgments. Ned Field and Seth Stein provided constructive reviews. This work was supported by NSF grants EAR-02-29834 and EAR-03-37226 and by a grant from the Southern California Earthquake Center.

\section{References}

Dieterich, J. H. (1994), A constitutive law for the rate of earthquake production and its application to earthquake clustering, J. Geophys. Res., 99 , 2601.

Fumal, T. E., R. J. Weldon, G. P. Biasi et al. (2002), Evidence for large earthquakes on the San Andreas Fault at the Wrightwood, Califormia, paleoseismic site: Ad 500 to present, Bull. Seismol. Soc. Am., 92, 2726. Harris, R. A., R. J. Archuletta, and S. M. Day (1991), Fault steps and the dynamic rupture process: $2-\mathrm{d}$ numerical simulations of a spontaneously propagating shear fracture, Geophys. Res. Lett., 18, 893.

King, G. C. P., and J. Nabelek (1985), The role of bends in faults in the initiation and termination of earthquake rupture: Implications for earthquake prediction, Science, $228,984$.

Lindh, A. G. (2003), Long-term earthquake forecasts in the San Francisco Bay area: A contrarian perspective, Eos Trans. $A G U, 84(46)$, Fall Meet. Suppl., Abstract NG41C-69.

Nishenko, S. P., and R. Buland (1987), A generic recurrence interval distribution for earthquake forecasting, Bull. Seismol. Soc. Am, 77, 1382.

Parsons, T., S. Toda, R. S. Stein et al. (2000), Heightened odds of large earthquakes near Istanbul: An interaction-based probability calculation, Science, 288, 661 .

Shaw, B. E. (1997), Model quakes in the two-dimensional wave equation, J. Geophys. Res., 102, 27,367.

Shaw, B. E. (2004), Self-organizing fault systems and self-organizing elastodynamic events on them: Geometry and the distribution of sizes of events, Geophys. Res. Lett., 31, L17603, doi:10.1029/2004GL019726.

Shaw, B. E., and J. R. Rice (2000), Existence of continuum complexity in the elastodynamics of repeated fault ruptures, J. Geophys. Res., 105, 23,791 .

Spyropoulos, C., C. H. Scholz, and B. E. Shaw (2002), Transition regimes for growing crack populations, Phys. Rev. $E, 65,56,105$.

Stein, S., and A. Newman (2004), Characteristic and uncharacteristic earthquakes as possible artifacts: Applications to the new Madrid and Wabash seismic zones, Seismol. Res. Lett., 75, 173.

Sykes, L. R. (2003), Long-term prediction of large earthquakes: When does quasi-periodic behavior occur?, Eos Trans. $A G U, 84(46)$, Fall Meet. Suppl., Abstract NG32A-01.

Working Group on California Earthquake Probabilities (2002), Earthquake probabilities in the San Francisco Bay region: 2002 to 2031, U.S. Geol. Surv. Circ., 1189.

B. E. Shaw, Lamont-Doherty Earth Observatory, Palisades, NY 10964, USA. (shaw@ideo.columbia.edu) 


\section{Electronic Supplement}

\section{Model Equations}

The equations of motion we are solving are as follows. In the $2 \mathrm{D}$ scalar bulk, we have

$$
\frac{\partial^{2} u}{\partial t^{2}}=\nabla^{2} u+(w-u)-\eta \frac{\partial u}{\partial t}+\nabla \cdot \mathbf{M}
$$

where $u$ is displacement, $t$ is time, $\nabla^{2}=\frac{\partial^{2}}{\partial x^{2}}+\frac{\partial^{2}}{\partial y^{2}}$ is the two-dimensional Laplace operator representing the horizontal elastic coupling of the displacement field, and the $w-u$ term represents the vertical coupling to the lower ductile layer. This layer is slowly stretched, loading the upper brittle layer and moving as

$$
w=\nu y t
$$

with $\nu \ll 1$. The dissipation constant $\eta$ damps the waves, and is used to mimic geometrical spreading effects which are otherwise much weaker in our 2D model as compared to 3D. The final term is the body forces arising from the fault dislocation openings $\mathbf{M}$

$$
\mathbf{M}=\left.\delta u\right|_{\Gamma}
$$

The boundary condition on the faults $\Gamma$ are that the normal strain equals the traction

$$
\left.\nabla u \cdot \perp \Gamma\right|_{\Gamma}=\phi .
$$

All of the nonlinearity in the problem is contained in the friction $\phi$, which has a stick-slip form, resisting motion up to some threshold value, and acting against motion when sliding occurs. We represent the stick-slip by

$$
\phi=\Phi\left(\frac{\partial S}{\partial t^{\prime}}, t^{\prime} \leq t\right) H\left(\frac{\partial S}{\partial t}\right)
$$

where $\Phi$ is a scalar frictional strength, $S=|\mathbf{M}|$ is the slip and $\partial S / \partial t$ is the slip rate on the fault, and $H$ is the antisymmetric step function

$$
H= \begin{cases}\frac{\partial S}{\partial t} & \frac{\partial S}{\partial t} \neq 0 \\ |H|<1 & \frac{\partial S}{\partial t}=0 .\end{cases}
$$

which represents the stick-slip nature of the friction, being multivalued at zero slip rate, and opposing motion in the $\hat{\partial S}$ unit direction when slipping.

What remains a big open question for earthquakes, is what is the frictional strength $\phi$. While there are reasons

Copyright 2003 by the American Geophysical Union.

Paper number .

$0094-8276 / 05 / \$ 5.00$ for thinking we may have a pretty good handle on what is happening at slow slip rates [Dieterich, 1994; Heslot et al., 1994], at high slip rates things are extremely uncertain, and many potential physical effects may be occurring, with substantially different implications for friction [Sibson, 1973; Melosh, 1996; Rice, 1999; Tullis and Goldsby, 2003]. With friction at high slip rates being an open question, we use a friction which has a minimum of parameters, is computationally efficient, and spans a range of frictional instabilities, including slip-, time-, and velocity- weakening [Shaw, 1995; Shaw and Rice, 2000]. Specifically, we use a $\Phi$ which combines long term geological strength $\Phi_{S}$ which weakens with accumulated geological slip [Spyropoulos et al., 2002] and a dynamic strength $\Phi_{Q}$ which weakens during events [Shaw, 1997]

$$
\Phi=\Phi_{S}+\Phi_{Q}
$$

The long term strength is given by

$$
\Phi_{S}=\Phi_{0}+\xi-\frac{\beta S}{1+a S}
$$

Here $\Phi_{0}$ is a constant overall strength which is irrelevant to the problem, $\xi$ is a random variable of amplitude between 0 and $\xi_{0}$, varying in space but fixed in time. This seeds some initial random strength heterogeneity in the model. Geological slip weakening occurs with the last term, which is proportional to slip $S$ with a constant $\beta$. $\beta$ affects the degree of localization in the problem, and therefore the resulting fault geometry. For large $\Phi_{0}$, we can operate in a regime where the saturating term $a$ is small and irrelevant. The brittle strain excess $\varepsilon \equiv\left(\nu t-\Phi_{0}\right) / \xi_{0}$ gives the relevant strain [Spyropoulos et al., 2002].

For the dynamic strength weakening, we consider three terms

$$
\Phi_{Q}=-\frac{\alpha Q}{1+\alpha Q}-\Sigma_{t}-\epsilon \nabla_{\|}^{2} \frac{\partial S}{\partial t}
$$

The first term, which is a function of heat $Q$, models frictional weakening from frictional heating; pore fluid effects [Sibson, 1973; Lachenbruch, 1980; Shaw, 1995] and flash heating of asperities [Rice, 1999] are two potentially relevant physical mechanisms which this simplified quantification could represent. The weakening rate constant $\alpha$ is a critical parameter in many aspects of the dynamics, although the results we present here are mainly insensitive to it. Heat accumulates with slip rate, and dissipates over some timescale $1 / \gamma$ :

$$
\frac{\partial Q}{\partial t}=-\gamma Q+\left|\frac{\partial S}{\partial t}\right| .
$$

Slip weakening results from $\gamma \ll 1$, while velocity weakening results from $\gamma \gg 1$ [Shaw, 1995; Shaw and Rice, 2000].

The second term in Equation (9)

$$
\Sigma_{t}= \begin{cases}\sigma_{0} \frac{t-t_{s}}{t_{0}} & t-t_{s}<t_{0} \\ \sigma_{0} & t-t_{s} \geq t_{0}\end{cases}
$$

is a nucleation term, which we make a big simplification of and consider as a time weakening term, which weakens with time $t$ over a timescale $t_{0}$ since beginning slipping at $t_{s}$ and 
restrengthens when resticking occurs. Th is allows for a huge numerical speedup compared with more expensive rate and state formulations, and the study of time weakening friction as well.

The last term $\epsilon \nabla_{\|}^{2} \frac{\partial S}{\partial t}$, with $\epsilon$ a small constant and $\nabla_{\|}^{2}$ the fault parallel second derivative, provides stability at the shortest wavelengths [Langer and Nakanishi, 1993; Shaw and Rice, 2000].

For numerical simplicity, we restrict the faults segments $\Gamma$ to be perpendicular to the stretching direction $y$. We also discretize the equations onto a rectangular grid, and use a second order finite difference approximation of the continuum equations. The numerical scheme proceeds by first evolving the fault system quasistatically, taking advantage of the dependence of the fault system evolution on the total slip, rather than slip increments, on the faults. Once a desired total strain is reached, the system is switched to elastodynamic mode. The system is loaded until one point is just at the point of failure. The event evolves then under fully inertial dynamics. Once the event has stopped slipping, the waves are quenched in the system, and the system is then reloaded until the next point is just at failure. Parameters used in the simulations shown, unless otherwise indicated, are: fault parameters $\beta=1.4, \varepsilon=1.0$; domain parameters $\delta_{x}=.125, \delta_{y}=.125, L_{x}=84, L_{y}=12$; bulk parameter $\eta=.3$; friction parameters $\alpha=3, \gamma=.1, \sigma_{0}=.3, t_{0}=.2$, $\epsilon=.003$.

\section{References}

Dieterich, J. H., A constitutive law for the rate of earthquake production and its application to earthquake clustering, $J$. Geo- phys. Res., 99, 2601, 1994.

Heslot, F., T. Baumberger, B. Perrin, B. Caroli, and C. Caroli, Creep, stick-slip, and dry-friction dynamics-experiments and a heuristic model, Phys. Rev. E, 49, 4973, 1994.

Lachenbruch, A., Frictional heating, fluid pressure, and the resistance to fault motion, J. Geophys. Res., 85, 6097, 1980.

Langer, J. S., and H. Nakanishi, Models of rupture propagation ii: Two dimensional model with dissipation on the fracture surface, Phys. Rev. E, 48, 439, 1993.

Melosh, H. J., Dynamical weakening of faults by acoustic fluidization, Nature, 379, 601, 1996.

Rice, J. R., Flash heating at asperity contacts and rate-dependent friction, Eos Than. $A G U$, 80, Abstract F681, 1999.

Shaw, B. E., Frictional weakening and slip complexity on earthquake faults, $J$. Geophys. Res., 100, 18,239, 1995.

Shaw, B. E., Modelquake in the two dimensional wave equation, J. Geophys. Res., 102, 27,367, 1997.

Shaw, B. E., and J. R. Rice, Existence of continuum complexity in the elastodynamics of repeated fault ruptures, $J$. Geophys. Res., 105, 23,791, 2000.

Sibson, R. H., Interactions between temperature and pore fluid pressure during earthquake faulting and a mechanism for partial or total stress relief, Nature Phys. Sci., 243, 66, 1973.

Spyropoulos, C., C. H. Scholz, and B. E. Shaw, Transition regimes for growing crack populations, Phys. Rev. E, 65, 056,105, 2002.

Tullis, T. E., and D. L. Goldsby, Flash melting of crustal rocks at almost seismic slip rates, Eos Tran. $A G U, 84$, Abstract S51B-05, 2003.

B. E. Shaw, Lamont-Doherty Earth Observatory, Palisades, NY 10964. (e-mail: shaw@ldeo.columbia.edu)

(Received 